Revue d'histoire de l'Amérique française

B REVUE D.HISTOIRE DE L'AMÉRIQUE FRANÇAISE

\title{
HÉBERT, Pierre, avec la collaboration de Marie-Pier LUNEAU, Lionel Groulx et L'appel de la race (Montréal, Fides, 1996), 204 p.
}

\section{Dominique Garand}

Volume 50, numéro 3, hiver 1997

URI : https://id.erudit.org/iderudit/305583ar

DOI : https://doi.org/10.7202/305583ar

Aller au sommaire du numéro

Éditeur(s)

Institut d'histoire de l'Amérique française

ISSN

0035-2357 (imprimé)

1492-1383 (numérique)

Découvrir la revue

Citer ce compte rendu

Garand, D. (1997). Compte rendu de [HÉBERT, Pierre, avec la collaboration de Marie-Pier LUNEAU, Lionel Groulx et L'appel de la race (Montréal, Fides, 1996), 204 p.] Revue d'histoire de l'Amérique française, 50(3), 458-462.

https://doi.org/10.7202/305583ar d'utilisation que vous pouvez consulter en ligne.

https://apropos.erudit.org/fr/usagers/politique-dutilisation/ 
HÉBERT, Pierre, avec la collaboration de Marie-Pier LUNEAU, Lionel Groulx et L'appel de la race (Montréal, Fides, 1996), 204 p.

Le but de cet ouvrage est exposé clairement dès la quatrième de couverture: par-delà le roman controversé de Groulx, il s'agit de présenter les lignes de force d'une époque à travers le parcours de l'un de ses plus illustres représentants. Après les polémiques que l'œuvre a suscitées, les auteurs ont choisi d'adopter un ton conciliant et un point de vue critique «neutre». Ils privilégient la présentation des faits et l'exposé de données empiriques à la discussion idéologique (trop souvent menée de façon stérile, il est vrai). $\mathrm{Ce}$ choix légitime n'empêche pas que transparaisse la sympathie qu'ils éprouvent, au départ, pour l'œuvre et la personne de Groulx. C'est elle qui détermine leur stratégie essayistique, malgré le souci apparent de peser le pour et 
le contre; une défense trop agressive ou partisane aurait en effet pour résultat de rendre les adversaires de Groulx sourds, dès le départ, à toute forme d'argumentation. Mais cet effort pour échapper à la polémique n'est pas sans conséquences, comme nous essayerons de le montrer un peu plus loin, la première étant de diluer quelque peu les enjeux au point d'affadir la question. Une seconde conséquence touche le traitement même de l'histoire littéraire, quand elle se réifie dans les statistiques.

L'essai procède de manière logique et chronologique. Le premier chapitre fournit des éléments biographiques en privilégiant les données relatives au parcours intellectuel et littéraire de Groulx. Ce portrait humanise la figure de Groulx, qui apparaît non pas comme un idéologue autoritaire, mais plutôt comme un idéaliste qui vivra ses instants d'enthousiasme, de désillusion et d'amertume. Le chapitre donne également une idée de l'époque du chanoine et de l'idéologie nationaliste, moins homogènes qu'on a tendance à le croire. C'est ainsi que l'on découvre un Groulx hostile aux prises de position de Duplessis et critiqué dans sa jeunesse par les autorités religieuses.

Le deuxième chapitre retrace les débuts littéraires de Groulx, qui publia en 1916 un recueil de récits et de souvenirs intitulé Les rapaillages. Les auteurs insistent sur l'influence de ce livre qui servit de modèle à de nombreuses publications régionalistes et fut réimprimé jusque dans les années quatre-vingt. Cette influence est indéniable, même si d'autres textes ont aussi concourru à l'élaboration de cette forme, comme Autour de la maison de Michelle Le Normand, paru la même année.

Le troisième chapitre aborde le rôle de Groulx dans le mouvement L'Action française, dont il fut le principal animateur (frôlant parfois l'omniprésence!). De nombreuses informations nous sont données au sujet de ce mouvement, de la revue qui le servait et de son entreprise éditoriale, sûrement la mieux articulée de son époque. Les stratégies éditoriales et les techniques de diffusion sont particulièrement bien étudiées. Le chapitre se clôt sur une analyse des procédés pseudonymiques de Groulx qui, on le sait avec certitude, en usa et abusa, pour des raisons qui relèvent à la fois de la censure ecclésiastique mais aussi, ne le cachons pas - mais justement, les auteurs de l'étude n'osent pas aller jusque-là —, d'une compulsion à tout contrôler caractéristique de la personnalité de Groulx. Ce trait est particulièrement apparent à l'époque de la publication de L'appel de la race, où sous divers pseudonymes on voit Groulx faire la promotion de son livre, donner des directives pour sa lecture, puis le défendre âprement contre les attaques auxquelles il s'est exposé. L'auteure de cette section de l'ouvrage a raison de noter: «Sans l'aide précieuse de ses doubles, comment Groulx aurait-il pu critiquer, à l'intérieur d'une revue placée sous sa direction, ses propres livres, parus, de surcroît, sous le label d'une maison d'édition qu'il contrôlait en partie?» (p. 82) Mais telle que posée, la question se présente comme une justification. C'est en de tels détours qu'une légère complaisance des auteurs se pointe, une manière subtile d'effacer les aspérités, non qu'il eût fallu soumettre Groulx à un procès moral, mais, tout de même, cette manière de jouer sur tous les fronts témoigne bien d'une posture intellectuelle, d'un 
certain rapport au pouvoir qui, eux, méritent d'être interrogés. Et à ce sujet, la comparaison avec Romain Gary ne tient pas le coup, car l'inflation pseudonymique chez Groulx n'a rien d'un jeu littéraire, le plus frappant étant que ses divers masques aient tous le même style!

Passons au quatrième chapitre, point d'aboutissement et raison d'être de l'ouvrage puisqu'il a pour objet L'appel de la race comme roman à thèse. L'analyse emprunte ici la voie des structures narratives, des thèmes et des motifs. Elle est menée avec un sens pédagogique exemplaire: elle s'amorce sur une description des divers états de l'avant-texte, elle enchaîne sur la reconstitution de la chronologie de la fiction racontée et de sa ponctuation narrative; suit une présentation des personnages, de leur caractérisation et de leur parcours narratif tel qu'il peut se représenter sur un schéma actantiel; l'auteur distingue ensuite les thèmes et les motifs du roman, voyant dans le «mariage mixte» et ses effets le thème dominant, ou structurant; suivent quelques remarques sur le réseau intertextuel dont est forgé L'appel de la race; la section suivante, sans doute la plus novatrice de l'étude, s'applique à identifier les passages où émerge la voix du narrateur, malgré son parti pris hétérodiégétique; enfin, il est fait état du genre dans lequel s'inscrit le roman, le roman à thèse, à partir des théories de S. Suleiman, dont l'auteur se réapproprie la distinction efficace entre structure d'apprentissage et structure antagonique (celle-ci caractérisant le roman à thèse), ce qui permet à Hébert de défendre l'hypothèse faisant de L'appel de la race un récit structuré antagoniquement, sauf dans son premier chapitre qui répond à la structure d'apprentissage (conclusion selon nous contestable dans la mesure où ce chapitre sert de fondement axiologique à la structure antagonique). En tout dernier lieu, la question du «racisme» du roman est étudiée, la conclusion de l'auteur étant que la notion de race chez Groulx n'induit pas un racisme ou une xénophobie mais participe plutôt d'une volonté d'être soi, de participer d'une filiation. Toutefois, l'auteur ne se demande pas comment de l'un on peut basculer dans l'autre, ou encore ce qui, chez Groulx, lui a permis justement de ne pas basculer, si tel est le cas.

Un dernier chapitre est consacré à la réception du livre. Plutôt court, ce chapitre constitue un bon résumé mais manque un peu de rigueur. Sans prétendre à l'exhaustivité, on aurait pu synthétiser de façon plus systématique les lectures qui ont été faites du roman, en faisant ressortir les enjeux soulevés sur les plans idéologique, esthétique et institutionnel. Au lieu de cela, les auteurs se contentent de faire état de la fortune, bonne ou mauvaise, du texte, en n'épinglant les tendances que de façon très générale. Cet exposé est complété par une étude des dédicaces offertes à Groulx. Ici encore, les critères d'analyse sont plutôt évasifs et il semble que l'exercice ait pour but principal d'attester du rayonnement de Groulx et de la reconnaissance obtenue auprès d'écrivains et d'intellectuels de toutes les générations et parfois assez éloignés de lui, comme si une telle observation tenait lieu d'argument. Les quelques dédicaces particulières qui sont observées ne conduisent qu'à des interprétations allusives, qui d'ailleurs laissent dans l'ombre toute théorisation de cette pratique ambiguë, nullement transparente et déterminée bien souvent par des contraintes mondaines et civiques. 
Ainsi, malgré l'excellence pédagogique que nous avons mentionnée, nous avons un peu à redire. Nos critiques ne touchent en rien la véracité des faits établis, des descriptions et observations sur le texte, mais plutôt la portée du regard analytique, l'éthique de la lecture, l'usage stratégique de l'histoire, bref, la conception des études littéraires qui se profile sous ce genre d'étude. La méthode utilisée dans l'ouvrage est souvent celle de la compilation et des statistiques. Pour éviter les débats idéologiques, l'auteur se rabat sur les données empiriques. Ainsi, Groulx est important, la preuve: il a laissé son nom à une station de métro, à un cégep, à des rues..., ses ouvrages ont été publiés à tant de milliers d'exemplaires, tous les intellectuels du Québec ont reconnu son importance comme en font foi les dédicaces, etc. Mais que l'influence de Groulx ait été déterminante, qui songerait à le nier? Le débat ne tourne pas autour de ce point mais autour du genre d'influence qu'il a eue; pour nous, il s'agit en premier lieu d'examiner le legs qu'il nous laisse: que vaut sa pensée pour nous aujourd'hui? dans quelle mesure son roman nous permet-il de penser notre situation et d'ouvrir le débat? Or, lorsqu'il est difficile d'actualiser un texte en exploitant en lui des éléments laissés jusquelà dans l'ombre, on se rabat sur l'historique et le sociologique: le texte est lu comme un témoignage, un document historique. C'est la voie choisie par l'auteur. Le nombre d'exemplaires vendus est certes à considérer, mais cela ne permet pas de conclure à l'importance d'une œuvre. À propos du succès des Rapaillages, Hébert signale furtivement, dans une note de bas de page (p. 48) que le livre a pu bénéficier d'une diffusion privilégiée dans le circuit des écoles, comme prix distribué aux élèves. Ce fait mériterait d'être souligné davantage car il en dit long sur le pouvoir régionaliste, lié pendant quelques décennies au pouvoir ecclésiastique et au pouvoir en matière d'éducation. Les rapaillages n'est pas un livre que l'on courait s'acheter chez le libraire, c'est un livre que des milliers d'élèves pendant des décennies se sont fait imposer par les autorités scolaires. La critique de J.-C. Harvey disant, dès 1925, que le livre est désuet (cité p. 39) n'est pas aussi absurde que l'auteur le laisse entendre: certes, d'un point de vue sociologique, il ne l'est pas puisqu'il est lu jusque dans les années quatre-vingt, mais Harvey est un écrivain qui adopte un point de vue esthétique et ce recueil est désuet, pourrait-on ajouter, pour la simple raison qu'il est presque impossible d'en renouveler la lecture, il est désuet parce que depuis les années vingt, il n'a pas suscité de nouvelle interprétation: c'est un livre déjà tout compris, qu'on ne consomme que pour le plaisir de la nostalgie.

Tel n'est pas le cas de L'appel de la race. Malheureusement, l'étude qu'en fait Hébert reste en-deçà de propositions qui ont déjà ouvert l'horizon interprétatif de ce roman. Il est certes intéressant d'établir en quoi ce texte répond à la structure du roman à thèse, quoique ce soit assez évident, mais que pense-t-on à partir de là, que fait-on de cette observation? Nous permetelle d'aller au-delà des commentaires moraux et esthétiques qui ont prédominé jusqu'à ce jour? Il est à notre avis beaucoup plus intéressant de lire ce texte dans son sémantisme structurant, ce qui permet d'établir, par exemple, que la question du mariage mixte n'est pas le cœur de l'ouvrage, mais une thématisation parmi d'autres d'un système de pensée qui oppose l'Identité et 
l'Aliénation, l'Unanimité et la Division, la Conservation et la Dégradation, la Reproduction et l'Entropie. Ces oppositions, en-deçà des prises de position, sont celles du discours régionaliste et commandent l'ensemble de leurs choix, quelles que soient les situations. On peut également ouvrir L'appel de la race en l'interprétant comme une histoire de généalogie et comme la recherche d'un nom propre perdu. On peut y lire la structure du fantasme qui ne trouve de satisfaction que dans l'imaginaire d'une fiction, malgré la prétention du texte à n'être pas littérature mais action. On peut circonscrire dans ce refus du geste littéraire l'indice de l'échec symbolique de Groulx, au sens où son combat aurait été miné de l'intérieur, aveugle à sa propre passion. Un tel jugement n'est en rien un rejet, il s'agit au contraire pour nous qui vivons aujourd'hui et sommes encore traversés par le discours groulxien, à la fois de l'historiciser, d'en reconnaître l'importance et de le réélaborer en connaissance de cause, d'en reprendre les signifiants (à l'intérieur desquels, pourquoi le nier, s'est formée notre identité collective) pour les déplacer. La lutte menée par Groulx nous touche très certainement, dans la mesure où elle s'articule sur des problèmes encore sensibles, mais il nous est possible de réfuter sa posture de sujet, telle qu'elle se donne à lire dans son roman, une fois établi qu'elle n'ouvre la voie qu'à un fantasme de souveraineté qui est un cul-de-sac mortifère conduisant tout droit au ressentiment ou à la mélancolie. Mais cette façon de lire un texte littéraire, libre et conséquente, qui engage le sujet de lecture tout autant que le sujet d'écriture, est rarement entendue.

L'essai d'Hébert et Luneau est donc prudent là où l'on eut souhaité plus d'audace, l'audace n'étant pas d'oser polémiquer mais de lire un livre au-delà de son vouloir-dire et d'ouvrir l'analyse à des voies inédites. Mais c'est peutêtre trop exiger des auteurs qui ont visé en premier lieu l'honnêteté intellectuelle, qualité que nous leur reconnaissons. Toutefois, sous des dehors d'objectivité et de neutralité, il n'échappe pas au lecteur que cet essai constitue une défense filiale de l'abbé Groulx, l'entreprise consistant maintenant à le justifier à l'aide d'arguments quantitatifs et institutionnels. Et la triste conséquence de ce refus de la mise en crise du «legs paternel» est d'embaumer le texte littéraire et de bloquer l'aventure intellectuelle. 